\title{
The role of BAM in mediating Fusobacterium nucleatum infection and pathogenesis \\ Claire Overly ${ }^{1}$, Nicholas Noinaj ${ }^{2}$ \\ ${ }^{1}$ Purdue University ${ }^{2}$ Purdue University \\ coverly@purdue.edu
}

Fusobacterium nucleatum is a Gram-negative oral pathogen implicated in periodontal infections and correlated with pre-term births and colorectal cancer. Additionally, this organism plays a vital role in the oral microbiome, adhering to other microbiome components through outer membrane proteins (OMPs) called adhesins. Multiple F. nucleatum adhesin structures have been identified as type Va autotransporters or porins, which are presented as $\beta$-barrel OMPs. The $\beta$-barrel assembly machinery (BAM) complex is an essential outer membrane protein complex found in all Gram-negative bacteria, functioning in the biogenesis of $\beta$-barrel OMPs. Components of this complex have been solved in organisms such as Escherichia coli, Haemophilus ducreyi, Neisseria gonorrhea, and Pseudomonas aeruginosa; however, the composition of the BAM complex in F. nucleatum remains unknown. In E. coli, the BAM complex is composed of an integral membrane protein, BamA, and four periplasmic lipoproteins, BamB-E. In F. nucleatum, only BamA appears to be present in the genome based on our bioinformatics analysis, despite the necessity of both BamA and BamD for organism viability in other Gram-negative bacteria. Since no BamD ortholog was found in F. nucleatum, we hypothesize that the BAM complex in F. nucleatum may use a different mechanism compared to E. coli. Thus, the goals of the project are first, to structurally characterize BamA in F. nucleatum using $\mathrm{X}$-ray crystallography and/or cryo-EM, and second, to determine the composition of the BAM complex in F. nucleatum by isolating and identifying BamA-interacting proteins. 